\title{
Extending and assessing composite electronic structure methods to the solid state
}

Cite as: J. Chem. Phys. 151, 121101 (2019); https://doi.org/10.1063/1.5123627

Submitted: 07 August 2019 . Accepted: 03 September 2019 . Published Online: 24 September 2019

L. Doná (D), J. G. Brandenburg (D), and B. Civalleri (D)
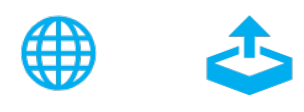

View Online

Export Citation

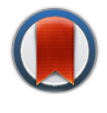

\section{ARTICLES YOU MAY BE INTERESTED IN}

Adventures in DFT by a wavefunction theorist

The Journal of Chemical Physics 151, 160901 (2019); https://doi.org/10.1063/1.5116338

Fantasy versus reality in fragment-based quantum chemistry

The Journal of Chemical Physics 151, 170901 (2019); https://doi.org/10.1063/1.5126216

Discovery of blue singlet exciton fission molecules via a high-throughput virtual screening and experimental approach

The Journal of Chemical Physics 151, 121102 (2019); https://doi.org/10.1063/1.5114789

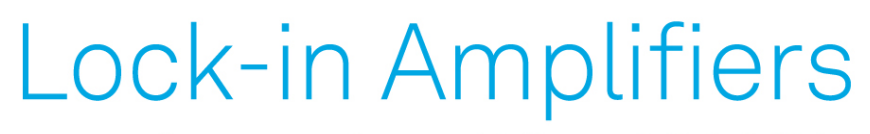

... and more, from DC to $600 \mathrm{MHz}$ Watch 


\title{
Extending and assessing composite electronic structure methods to the solid state
}

\author{
Cite as: J. Chem. Phys. 151, 121101 (2019); doi: 10.1063/1.5123627 \\ Submitted: 7 August 2019 - Accepted: 3 September 2019 • \\ Published Online: 24 September 2019
}

\section{Doná, (D) J. G. Brandenburg, ${ }^{2, a)}$ (D) and B. Civalleri ${ }^{1, b)}$ (D)}

\begin{abstract}
AFFILIATIONS
${ }^{1}$ Dipartimento di Chimica, Università di Torino and NIS (Nanostructured Interfaces and Surfaces) Centre, Via P. Giuria 5, 10125 Torino, Italy

${ }^{2}$ Interdisciplinary Center for Scientific Computing, University of Heidelberg, Im Neuenheimer Feld 205A, 69120 Heidelberg, Germany
\end{abstract}

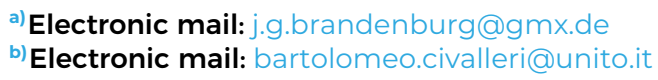

\begin{abstract}
A hierarchy of simplified Hartree-Fock (HF), density functional theory (DFT) methods, and their combinations has been recently proposed for the fast electronic structure computation of large systems. The covered methods are a minimal basis set Hartree-Fock (HF-3c), a small basis set global hybrid functional (PBEh-3c), and its screened exchange variant (HSE-3c), all augmented with semiclassical correction potentials. Here, we extend their applicability to inorganic covalent and ionic solids as well as layered materials. The new methods have been dubbed HFsol-3c, PBEsol0-3c, and HSEsol-3c, respectively, to indicate their parent functional as well as the correction potentials. They have been implemented in the CRYsTAL code to enable routine application for molecular as well as solid materials. We validate the new methods on diverse sets of solid state benchmarks that cover more than 90 solids ranging from covalent, ionic, semi-ionic, layered, and molecular crystals. While we focus on structural and energetic properties, we also test bandgaps, vibrational frequencies, elastic constants, and dielectric and piezoelectric tensors. HSEsol-3c appears to be most promising with mean absolute error for cohesive energies and unit cell volumes of molecular crystals of $1.5 \mathrm{kcal} / \mathrm{mol}$ and $2.8 \%$, respectively. Lattice parameters of inorganic solids deviate by $3 \%$ from the references, and vibrational frequencies of $\alpha$-quartz have standard deviations of $10 \mathrm{~cm}^{-1}$. Overall, this shows an accuracy competitive to converged basis set dispersion corrected DFT with a substantial increase in computational efficiency.
\end{abstract}

Published under license by AIP Publishing. https://doi.org/10.1063/1.5123627

\section{INTRODUCTION}

Kohn-Sham density functional theory (DFT) is routinely used for the fast computation of large systems and will most likely continue to be the method of choice for the generation of reliable geometries in the foreseeable future. ${ }^{1,2}$ Recently, a hierarchy of simplified electronic structure methods designed for consistent structures and noncovalent interactions of large systems has been developed on the past five years. ${ }^{3}$ They are designed as an ideal compromise between cost and accuracy for calculations on molecular systems of increasing size. Successful applications include protein-ligand binding affinities, ${ }^{4}$ large molecular crystals with shortest intermolecular hydrogen contacts, ${ }^{5}$ unusual halogen bonding motifs, ${ }^{6}$ and screening of zeolite thermodynamics. ${ }^{7}$ They are based on the pure Hartree-Fock (HF) method or HF/DFT hybrid functionals with the target of yielding good structures and reasonable energetic properties. The key ingredients are (i) the use of minimal or small-to-medium basis sets expressed in terms of atomcentered Gaussian-type functions and (ii) the combination of three (or two) semiclassical atom-pairwise (or triplewise) corrections to include London dispersion interactions, ${ }^{8-10}$ to remove the basis set superposition error (BSSE), ${ }^{11}$ and to compensate for the basis set incompleteness error (BSIE) through a short-range (SRB) correction. This has led to a pseudohierarchical ladder of methods in which the percentage of Hartree-Fock exchange ranges from 100\% (i.e., full $\mathrm{HF}$ ) to $0 \%$ [i.e., pure generalized gradient approximated (GGA) functional] passing through $42 \%$ for hybrid HF/DFT functionals and the basis set size increases from minimal to double-zeta and to triplezeta quality. The resulting methods have been dubbed as $\mathrm{HF}-3 \mathrm{c},{ }^{12}$ PBEh-3c, ${ }^{13}$ HSE-3c, ${ }^{14}$ and B97-3c. ${ }^{1}$ 
The four methods have been successfully applied to study small-to-large molecules and molecular adducts and have been extended to periodic systems, in particular, to study molecular crystals. ${ }^{13-16}$ Although the composite methods cover all elements of the periodic table, their application to inorganic solids is mainly limited by the adoption of molecular basis sets. Unmodified molecular basis sets can be problematic to use in certain solid state calculations ${ }^{17-19}$ because they usually contain basis functions with low exponents that can lead to numerical instability and linear dependency problems.

In this communication, we extend the applicability of three composite methods to inorganic solids and layered materials. Our guidelines for the revisions (i) employ exchange-correlation functionals developed for solids (i.e., $\mathrm{PBEsol}^{20}$ and $\mathrm{HSEsol}^{21}$ ), (ii) reduce the amount of HF exchange in DFT hybrid methods for a better description of electronic properties ${ }^{22-24}$ (e.g., 25\%), and (iii) apply a simple recipe to make molecular basis sets suitable for inorganic solids. The revised methods have been tagged with a label "sol" (as for "solids") to distinguish them from the original ones and are thus denoted as HFsol-3c, PBEsol0-3c, and HSEsol-3c.

\section{COMPUTATIONAL METHODS}

\section{A. Methodologies}

The total energy provided by the composite methods can be written as

$$
E_{\mathrm{tot}}^{\mathrm{sol}-3 \mathrm{c}}=E_{\mathrm{tot}}^{\mathrm{HF}, \mathrm{DFT} / \mathrm{basis}}+E_{\mathrm{disp}}^{\mathrm{D} 3}+E_{\mathrm{BSSE}}^{\mathrm{gCP}}+E^{\mathrm{SRB}} .
$$

The different contributions are discussed in detail below. $E_{\text {tot }}^{\mathrm{HF}, \mathrm{DFT} / \mathrm{basis}}$ denotes the total energy evaluated at the HF or DFT level of theory in a fixed basis set expansion. In the present work, we use HF, the global hybrid functional PBEsol0, ${ }^{20}$ and the screened exchange hybrid functional HSEsol. ${ }^{21}$ The latter two are chosen because they were specifically devised for solids. We revise the original MINIX basis set for HFsol-3c and def2-mSVP for PBEsol0-3c and HSEsol-3c (see later on for details). The total energy is supplemented with an established semiclassical London dispersion correction (D3 model). ${ }^{2}$ D3 is used in the rational (Becke-Johnson) damping variant ${ }^{26}$ and includes dipole-dipole, dipole-quadrupole, as well as three-body triple-dipole terms. While the long-range contributions are determined by the $a b$ initio computed dispersion coefficients, the shortrange damping includes two empirically optimized parameters. The removal of the BSSE due to the use of small basis sets with large BSIE is accomplished through a geometrical counterpoise correction (gCP). A precomputed element and basis set specific BSIE measure is used to generate a repulsive atom-pairwise potential with four free parameters. HF-3c employs an additional short-range basis set (SRB) correction that corrects the systematic overestimation of bond lengths involving electronegative elements. The two SRB parameters are trained on a set of 107 small molecule structures. ${ }^{12} \mathrm{~A}$ comparison of the method hierarchy with different ingredients is given in Table I. All functional and basis set specific parameters were empirically optimized for each revised composite method as shortly discussed in the Appendix, and their values are reported in Table IV.
TABLE I. Feature summary of the revised composite methods.

\begin{tabular}{lccc}
\hline \hline & HFsol-3c & PBEsol0-3c & HSEsol-3c \\
\cline { 2 - 4 } Method & HF & PBEsol0 & HSEsol \\
\hline AO basis set & sol-MINIX & sol-def2-mSVP & sol-def2-mSVP $^{2}$ \\
HF exchange $\%$ & 100 & 25 & $25-0^{\text {a }}$ \\
D3 dispersion & Yes & Yes & Yes \\
gCP correction & Yes & Yes & yes \\
SRB correction & Yes & No & No \\
\hline \hline
\end{tabular}

${ }^{\mathrm{a}} 25 \%$ at short-range and $0 \%$ at long-range using standard range-separation with $\omega=0.11$.

${ }^{\mathrm{b}}$ Damped variant of gCP correction is used. ${ }^{13}$

\section{B. Basis set revision}

The original composite methods use minimal (MINIX) and double-zeta quality (def2-mSVP) atomic basis sets for HF and hybrid DFT, respectively. As previously mentioned, they are based on molecular basis sets that are not fully suitable for certain solid state calculations. Therefore, one of the main purposes of the present work was a revision of these basis sets. To that aim, we applied a simple recipe based on a rescaling of the exponents of the outermost uncontracted Gaussian functions. For sake of brevity, more details about the revision of the basis sets are reported in the supplementary material.

Basis set exponents have been revised from He to Xe for def2mSVP. ${ }^{27}$ We follow the two steps:

(i) Scale the exponent of the most diffuse Gaussian function to a value equal or slightly greater than $0.1 \mathrm{Bohr}^{-2}$ that has been considered as a lower bound limit to avoid numerical instability.

(ii) Scale the exponent of the previous Gaussian function by keeping the original exponent ratio.

Steps (i) and (ii) of this scheme were applied to $s$ and $p$ shells, while for $d$ and $f$ functions solely, step (i) was applied. For the MINIX basis set, the same procedure is adopted, where for elements $\mathrm{H}-\mathrm{Ar}$, the orbitals are decontracted before applying the scaling. The revised basis sets have been implemented in CRYSTAL17 and are explicitly given in the supplementary material.

\section{Computational details}

The revised composite methods for solids have been implemented in a development version of the CRYSTAL17 code. ${ }^{17,28}$ The crystalline orbitals are represented as linear combinations of Bloch functions, with each of them being built from atom-centered atomic orbitals (AO), which are expressed in terms of Gaussiantype-functions. In contrast to plane-wave codes, the implementation of Fock exchange is easier in terms of $\mathrm{AO}$ and well-established in CRYSTAL since more than three decades. ${ }^{29}$ CRYSTAL is the ideal program for large scale solid state applications as it can employ all point and space group symmetries. ${ }^{30}$ Furthermore, it scales well on high-performance computational facilities with up to 30000 cores, and electronic structure calculations on 14000 atoms in the primitive unit cell have been presented recently. ${ }^{31}$ All calculations for 
TABLE II. Statistical analysis of original and revised composite methods on the benchmark set S66 $\times 8^{32}$ and $X 23.33,34$

\begin{tabular}{lcccccccc}
\hline \hline Dataset & Prop. & & HF-3c & HFsol-3c & PBEh-3c & PBEsol0-3c & HSE-3c & HSEsol-3c \\
\hline S66x8 $^{\text {a }}$ & Dist. & MARE (\%) & 0.50 & 0.39 & 1.50 & 0.51 & 1.50 & 0.49 \\
& BE & MAE (kcal/mol) & 0.43 & 0.71 & 0.50 & 0.64 & 0.50 & 0.66 \\
$\mathrm{X}_{23} 3^{\mathrm{b}}$ & Vol. & MARE (\%) & 6.46 & 2.31 & 3.60 & 3.18 & 2.90 & 2.84 \\
& CE & MAE (kcal/mol) & 2.06 & 3.03 & 1.30 & 1.53 & 1.30 & 1.50 \\
\hline \hline
\end{tabular}

${ }^{a}$ Equilibrium distance (Dist.) and binding energy (BE). This set was part of the D3 and gCP parameter training.

${ }^{\mathrm{b}}$ Equilibrium unit cell volume (Vol.) and cohesive energy (CE).

TABLE III. Mean absolute error of original and revised composite methods on the benchmark set SS20.22

\begin{tabular}{lrccccc}
\hline \hline Property $^{\mathrm{a}, \mathrm{b}}$ & HF $^{\mathrm{b}}$ & HFsol-3c $^{\mathrm{b}}$ & PBEsol0 $^{\mathrm{b}}$ & PBEsol0-3c $^{\mathrm{b}}$ & HSEsol $^{\mathrm{b}}$ & HSEsol-3c $^{\mathrm{b}}$ \\
\hline LP $(\AA)$ & 0.07 & 0.07 & 0.03 & 0.03 & 0.03 & 0.03 \\
BG $(\mathrm{eV})$ & 6.75 & 6.95 & 0.78 & 0.92 & 0.67 & 0.77 \\
BM $(\mathrm{GPa})$ & 22.05 & 26.70 & 9.34 & 7.93 & 8.96 & 7.63 \\
\hline \hline
\end{tabular}

${ }^{\text {a }}$ Lattice parameter (LP), bandgap (BG), and bulk modulus (BM).

b Used in identical basis set expansion as corresponding " $3 \mathrm{c}$ " methods, but without correction potentials.

both revision and validation purposes were carried out with default computational parameters.

\section{RESULTS AND DISCUSSION}

The performance of the revised composite methods was first benchmarked against corresponding results of the original methods for the S66x8 dataset of molecular dimers ${ }^{32}$ and the X23 set of molecular crystals. ${ }^{33,34}$ In addition, a set of 20 simple inorganic solids ${ }^{22}$ with cubic structure, dubbed as SS20, was used to compare them with the uncorrected methods (i.e., HF, PBEsol0, and HSEsol). Here, lattice constants, bandgaps, and bulk moduli are tested. Lattice parameters have been corrected to remove thermal and zeropoint effects. ${ }^{35}$ Statistical results of original and revised composite methods are given in Tables II and III.

For the noncovalently interacting systems S66 and X23, the uncorrected methods do not yield satisfactory results. All composite methods yield binding energies and equilibrium geometries competitive to results of state-of-the-art dispersion corrected DFT (see Table 9 of Ref. 10). The revised (sol) variants significantly improve the dimer distances and unit cell volumes of molecular crystals while just slightly deteriorating the binding and cohesive energies, respectively. In particular, HSEsol-3c seems to be promising with a mean absolute error (MAE) of $1.5 \mathrm{kcal} / \mathrm{mol}$ and a mean absolute relative error in percentage [MARE (\%)] of $2.8 \%$ for the X23 lattice energies and unit cell volumes, respectively. Keeping in mind the minimal basis set leading to a substantial speed-up, HF-3c and HFsol-3c results are also satisfying (see also Ref. 16 for broader molecular crystal tests). Overall, the reparameterization keeps the excellent accuracy of the original composite methods for noncovalently bound systems, which is mandatory if the revised methods are intended as generally applicable methods.
The original composite methods could not be tested on the SS20 set because the SCF is not converging within standard settings. In contrast, the revised methods all converged smoothly, which is important for a readily applicable method. Figure 1 shows the correlation between computed and experimental lattice parameters. As summarized in Table III, all three revised methods give excellent results with MAE well below $0.1 \AA$. Results from PBEsol0-3c and HSEsol-3c are almost identical, demonstrating that the long-range Fock exchange is not needed for the properties under consideration. Not unexpectedly, bandgaps are substantially overestimated by HFsol-3c, whereas hybrid composite methods for solids perform much better with a MAE below $1 \mathrm{eV}$, which is close to uncorrected

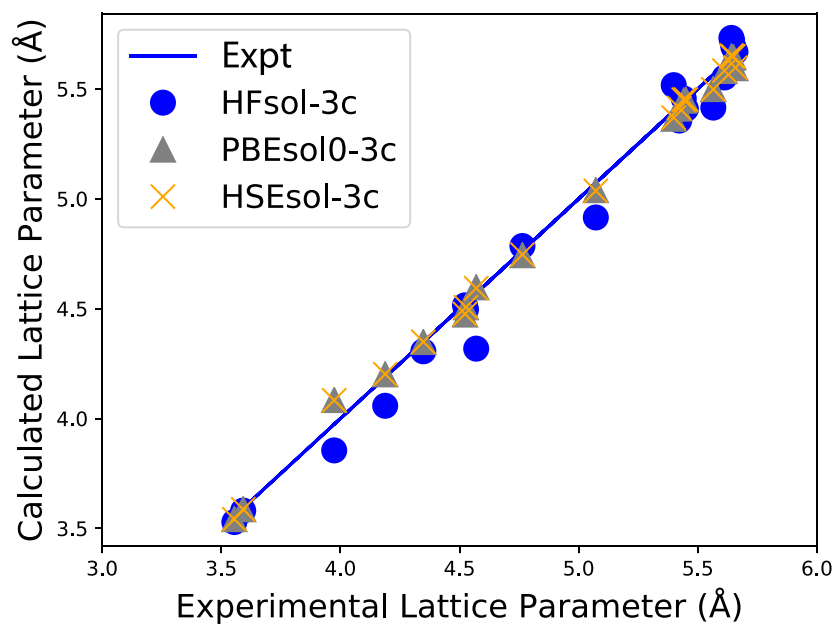

FIG. 1. Lattice parameters for the SS20 dataset computed with the revised composite methods compared to experimental reference data. 
functionals. The same holds for the computed bulk moduli with the accuracy being slightly improved by the correction potentials.

We additionally considered a broader set of more than 50 different inorganic solids containing cubic, hexagonal, tetragonal, and orthorhombic, ionic, and covalent systems (results summarized in the supplementary material). ${ }^{36,37}$ While HFsol-3c does not yield reasonable lattice parameters, PBEsol0-3c and HSEsol-3c have MAREs of $3.3 \%$ and $3.4 \%$, respectively, which is similar to typical hybrid functionals like PW1PW evaluated in larger triple-zeta basis set expansions. Overall, the revised methods can be successfully applied to inorganic solids, and, in particular, the two hybrid methods give results of triple-zeta quality with significantly reduced computational cost. For instance, a HSEsol-3c calculation on a 64-atom NiO supercell is more than twice as fast compared to the same functional with a pob-TZVP basis set. See the supplementary material for further data.

Layered materials are challenging systems because of the combination of strong covalent bonds (intralayer) and weak van der Waals interactions (interlayer). Here, we test graphite, hexagonal BN (h-BN), and black phosphorus (black-P) for which high level theoretical reference data on the binding energy as well as high-quality experimental data on structural features are available. When compared to the uncorrected methods, all revised composite methods provide good structural properties and interlayer energies. However, the performance seems to be very system specific. For graphite, the three revised methods provide good results, in particular, HFsol-3c, while the two hybrid methods give slightly underestimated interlayer energies. The opposite is observed in the case of h-BN. All three methods predict an interlayer energy in good agreement with the diffusion Monte Carlo (DMC), ${ }^{38}$ while the interlayer lattice parameter tends to be underestimated, although the experimental lattice parameters $^{39}$ were not back-corrected to the athermal limit. Finally, for black-P, the interlayer distance is underestimated by the two hybrids but slightly overestimated by HFsol-3c. In turn, the interlayer energy (in meV/atom) computed with PBEsol0-3c and HSEsol$3 c$ ( -163 and 164, respectively) results to be substantially overestimated compared to the DMC and coupled-cluster single double triple coupled cluster singles-and doubles including perturbative triple excitations $[\mathrm{CCSD}(\mathrm{T})]$ estimates $(-80$ and -92 , respectively). The problematic exfoliation energy of black-P seems to be related to the damping function of the used dispersion correction as analyzed in Ref. 40. Incidentally, HFsol-3c (-88) is in excellent agreement with reference data. ${ }^{40,4}$

Other important properties of solids are vibrational frequencies, elastic, dielectric, and piezoelectric properties. We tested the revised composite methods on $\alpha$-quartz (see the supplementary material). ${ }^{42-45}$ As expected, HF in a MINIX basis set is not capable of describing these properties. On the other hand, PBEsol0-3c and HSEsol-3c give results in good agreement with the experiment. Especially, vibrational frequencies have small errors with a MAE of about $8 \mathrm{~cm}^{-1}$ and a standard deviation of $10 \mathrm{~cm}^{-1}$. Notably, for the latter methods, the cost of the calculation is about 3 times less expensive than with a triple-zeta basis set as pob-TZVP.

\section{CONCLUSIONS AND PERSPECTIVES}

In this work, we have presented a revision of the composite methods pioneered by Grimme and co-workers ${ }^{12-14}$ that were originally designed with specific focus on consistent structures and interactions of large molecular systems and molecular crystals. Here, we changed the adopted semilocal functional, revised the basis set expansions, and refitted the D3 and gCP corrections. The revised methods, dubbed as "sol-3c" to emphasize the focus on solids, were benchmarked on different datasets including molecular adducts, molecular crystals, and a large set of solids including covalent, ionic, semi-ionic, and layered materials. Different properties were tested from structures to energetics, from bandgaps to vibrational frequencies and response properties. Overall, the results demonstrate that the revised methods perform equally well as the original ones and importantly extend and improve their applicability significantly.

As expected, HFsol-3c shows some drawbacks because of the known limitations of plain HF and the minimal basis set. We expect that the best results can be obtained when dealing with molecular crystals for which structure and energetics are strikingly accurate. For covalent and ionic solids, the revised HFsol-3c shows a moderately good accuracy for structural features, which can be sufficient for screening applications. Known deficiencies of HF limit the use of HFsol-3c for metals and very small-gap systems.

On the other hand, composite methods based on hybrid functionals for solids and a double-zeta quality basis set (i.e., PBEsol0-3c and HSEsol-3c) show consistently good performance over all system classes, and the structural, chemical, and physical properties of solids are very well described. We expect that they can also be safely applied to small-gap and metallic systems, in particular, HSEsol-3c, which is based on a screened Coulomb exchange functional. They are overall well-suited for a broad range of applications in solid state chemistry and physics.

Work is in progress to apply these methods to porous materi$\mathrm{als}^{7}$ and metal-organic frameworks. The accuracy of hybrid composite methods combined with their computational efficiency is ideal for high-throughput screenings. Further improvements can be foreseen by the inclusion of the D4 dispersion correction ${ }^{46,47}$ or a more specific refitting of the parameters based on solid state reference data.

\section{SUPPLEMENTARY MATERIAL}

Details of the revision of the def2-SVP and MINIX basis sets and the modified basis sets in CRYSTAL format, all computed data for the X23, SS20, and POB datasets and results for the structure, vibrational frequencies, elastic, dielectric, and piezoelectric properties of $\alpha$-quartz are included in the supplementary material.

\section{ACKNOWLEDGMENTS}

L.D. thanks Angelos Michaelides for hosting him at University College London (UK) to work with J. Gerit Brandenburg. J.G.B. acknowledges support from the Alexander von Humboldt foundation.

\section{APPENDIX: SEMICLASSICAL CORRECTIONS AND REFITTING}

Revised composite methods required a careful reparameterization because of the different exchange-correlation functionals adopted and the basis sets' revision. Therefore, both D3 and the gCP 
TABLE IV. Summary of all empirical parameters of the revised composite methods.

\begin{tabular}{|c|c|c|c|c|c|c|c|}
\hline & \multicolumn{3}{|c|}{ D3 } & \multicolumn{4}{|c|}{$\mathrm{gCP}$} \\
\hline & $a_{1}$ & $a_{2}$ & $s_{8}$ & $\alpha$ & $\beta$ & $\sigma$ & $\eta$ \\
\hline HSEsol-3c & 0.520 & 4.939 & $0.000^{\mathrm{a}}$ & 0.294 & 1.957 & $1.000^{\mathrm{a}}$ & 1.428 \\
\hline PBEsol0-3c & 0.536 & 4.645 & $0.000^{\mathrm{a}}$ & 0.275 & 1.965 & $1.000^{\mathrm{a}}$ & 1.369 \\
\hline \multirow[t]{3}{*}{ HFsol-3c } & 0.417 & 2.915 & 0.237 & 1.155 & 1.176 & 0.129 & 1.153 \\
\hline & & & & & $\mathrm{SRB}$ & $\mathrm{s}$ & $\gamma$ \\
\hline & & & & & & $0.03^{b}$ & $0.70^{\mathrm{b}}$ \\
\hline
\end{tabular}

${ }^{\mathrm{a}}$ Value not optimized.

${ }^{\mathrm{b}}$ Value taken from original HF-3c parameterization.

specific parameters were reoptimized by using the S66x8 dataset. ${ }^{3}$ In order to achieve more flexibility with the $\mathrm{D} 3 / \mathrm{gCP}$ corrections, the fitting procedure was applied simultaneously to the $\mathrm{D} 3$ and gCP part.

For PBEsol0-3c and HSEsol-3c methods, we redetermined the parameters in the Becke-Johnson damping function for the D3 correction,

$$
E_{\text {disp }}^{\mathrm{D} 3}=-\frac{1}{2} \sum_{A B} \sum_{n=6,8} s_{n} \frac{C_{n}^{A B}}{R_{A B}{ }^{n}} f_{\text {damp }}^{(n)}\left(R_{A B}\right) .
$$

Here, $C_{n}^{A B}$ denotes the $n$ th-order dispersion coefficient (orders $=6$, 8) for each atom pair $\mathrm{AB}, R_{A B}$ is their internuclear distances, and $s_{n}$ are the order-dependent scaling factors. The rational Becke-Johnson damping function is

$$
f_{\text {damp }}^{(n)}\left(R_{A B}\right)=\frac{R_{A B}^{n}}{R_{A B}^{n}+\left(a_{1} R_{0}^{A B}+a_{2}\right)^{n}} .
$$

The damping function incorporates radii for atomic pairs $R_{0}^{A B}$ $=\sqrt{C_{8}^{A B} / C_{6}^{A B}}$ and functional-specific parameters $a_{1}$ and $a_{2}$ that have been refitted in the present work. In addition, the AxilrodTeller-Muto $^{48,49}$ (ATM) three-body dipole-dipole-dipole term is also included.

The gCP correction is given by the atom-pairwise potential,

$$
E_{\mathrm{BSSE}}^{\mathrm{gCP}}=\frac{\sigma}{2} \sum_{A B} V_{A}^{\mathrm{gCP}}\left(R_{A B}\right) f_{\mathrm{damp}}^{\mathrm{gCP}}\left(R_{A B}\right)
$$

The difference in atomic energy between a large (nearly complete) basis set and the target basis set for each free atom is used as a measure to generate the repulsive potential $V_{A}^{\mathrm{gCP}}$ with fitting parameters $\alpha, \beta$, and $\eta$. As originally proposed for the PBEh- $3 \mathrm{c}$ and HSE- $3 \mathrm{c}$ methods, the value of $\sigma, s_{6}$, and $s_{8}$ was fixed to $1.00,1.00$, and 0.00 , respectively.

Note that for the D3 correction in HFsol-3c [see Eq. (A1)], we only modified the $s_{8}$ scaling factor reducing it by a factor of 0.7 as proposed for the original HF-3c method in Ref. 16, all other parameters were unchanged. The parameters of the short range correction for $\mathrm{HF}$ were not re-evaluated, and gCP is used in its undamped variant.

The whole sets of refitted values for the three revised composite methods are reported in Table IV.

\section{REFERENCES}

${ }^{1} \mathrm{~K}$. Burke, "Perspective on density functional theory," J. Chem. Phys. 136(15), 150901 (2012).

${ }^{2}$ R. J. Maurer, C. Freysoldt, A. M. Reilly, J. G. Brandenburg, O. T. Hofmann, T. Bjöorkman, S. Lebègue, and A. Tkatchenko, "Advances in density-functional calculations for materials modeling," Annu. Rev. Mater. Res. 49, 1-30 (2019).

${ }^{3}$ E. Caldeweyher and J. G. Brandenburg, "Simplified DFT methods for consistent structures and energies of large systems," J. Phys.: Condens. Matter 30, 213001 (2018).

${ }^{4}$ S. Ehrlich, A. H. Göller, and S. Grimme, "Towards full quantum-mechanicsbased protein-ligand binding affinities," ChemPhysChem 18, 898-905 (2017).

${ }^{5}$ S. Rösel, H. Quanz, C. Logemann, J. Becker, E. Mossou, L. Cañadillas-Delgado, E. Caldeweyher, S. Grimme, and P. R. Schreiner, "London dispersion enables the shortest intermolecular hydrocarbon $\mathrm{H} \cdots \mathrm{H}$ contact," J. Am. Chem. Soc. 139(22), 7428-7431 (2017).

${ }^{6}$ T. A. Schaub, R. Sure, F. Hampel, S. Grimme, and M. Kivala, "Quantum chemical dissection of the shortest $\mathrm{P}=\mathrm{O} \cdots \mathrm{I}$ halogen bond: The decisive role of crystal packing effects," Chem. Eur. J. 23(24), 5687-5691 (2017).

${ }^{7}$ M. Cutini, B. Civalleri, and P. Ugliengo, "Cost-effective quantum mechanical approach for predicting thermodynamic and mechanical stability of pure-silica zeolites," ACS Omega 4, 1838-1846 (2019).

${ }^{8} \mathrm{~J}$. Klimeš and A. Michaelides, "Perspective: Advances and challenges in treating van der Waals dispersion forces in density functional theory," J. Chem. Phys. 137, 120901 (2012).

${ }^{9}$ K. Berland, V. R. Cooper, K. Lee, E. Schröder, T. Thonhauser, P. Hyldgaard, and B. I. Lundqvist, "van der Waals forces in density functional theory: A review of the vdW-DF method," Rep. Prog. Phys. 78, 066501 (2015).

${ }^{10}$ S. Grimme, A. Hansen, J. G. Brandenburg, and C. Bannwarth, "Dispersioncorrected mean-field electronic structure methods," Chem. Rev. 116, 5105-5154 (2016).

${ }^{11} \mathrm{H}$. Kruse and S. Grimme, "A geometrical correction for the inter-and intramolecular basis set superposition error in Hartree-Fock and density functional theory calculations for large systems," J. Chem. Phys. 136, 154101 (2012).

${ }^{12} \mathrm{R}$. Sure and S. Grimme, "Corrected small basis set Hartree-Fock method for large systems," J. Comput. Chem. 34, 1672-1685 (2013).

${ }^{13}$ S. Grimme, J. G. Brandenburg, C. Bannwarth, and A. Hansen, "Consistent structures and interactions by density functional theory with small atomic orbital basis sets," J. Chem. Phys. 143, 054107 (2015).

${ }^{14}$ J. G. Brandenburg, E. Caldeweyher, and S. Grimme, "Screened exchange hybrid density functional for accurate and efficient structures and interaction energies," Phys. Chem. Chem. Phys. 18, 15519-15523 (2016).

${ }^{15}$ J. G. Brandenburg, C. Bannwarth, A. Hansen, and S. Grimme, "B97-3c: A revised low-cost variant of the B97-D density functional method," J. Chem. Phys. 148, 064104 (2018).

${ }^{16}$ M. Cutini, B. Civalleri, M. Corno, R. Orlando, J. G. Brandenburg, L. Maschio, and P. Ugliengo, "Assessment of different quantum mechanical 
methods for the prediction of structure and cohesive energy of molecular crystals," J. Chem. Theory Comput. 12, 3340-3352 (2016).

${ }^{17}$ R. Dovesi, V. R. Saunders, C. Roetti, R. Orlando, C. M. Zicovich-Wilson, F. Pascale, B. Civalleri, K. Doll, N. M. Harrison, I. J. Bush, P. D'Arco, M. Llunell, M. Causà, Y. Noël, L. Maschio, A. Erba, M. Rerat, and S. Casassa, CRYSTAL17 (Universitá di Torino, Torino, 2017).

${ }^{18}$ A. Grüneich and B. A. Heß, "Choosing GTO basis sets for periodic HF calculations," Theor. Chem. Acc. 100, 253-263 (1998).

${ }^{19} \mathrm{~F}$. Jensen, "Analysis of energy-optimized Gaussian basis sets for condensed phase density functional calculations," Theor. Chem. Acc. 132, 1380 (2013).

${ }^{20}$ J. P. Perdew, A. Ruzsinszky, G. I. Csonka, O. A. Vydrov, G. E. Scuseria, L. A. Constantin, X. Zhou, and K. Burke, "Restoring the density-gradient expansion for exchange in solids and surfaces," Phys. Rev. Lett. 100, 136406 (2008).

${ }^{21}$ L. Schimka, J. Harl, and G. Kresse, "Improved hybrid functional for solids: The HSEsol functional," J. Chem. Phys. 134, 024116 (2011).

${ }^{22}$ P. Pernot, B. Civalleri, D. Presti, and A. Savin, "Prediction uncertainty of density functional approximations for properties of crystals with cubic symmetry," J. Phys. Chem. A 119, 5288-5304 (2015).

${ }^{23} \mathrm{~J}$. M. Crowley, J. Tahir-Kheli, and W. A. Goddard III, "Resolution of the band gap prediction problem for materials design," J. Phys. Chem. Lett. 7, 1198-1203 (2016).

${ }^{24}$ A. J. Garza and G. E. Scuseria, "Predicting band gaps with hybrid density functionals," J. Phys. Chem. Lett. 7, 4165-4170 (2016).

${ }^{25}$ S. Grimme, J. Antony, S. Ehrlich, and H. Krieg, "A consistent and accurate $a b$ initio parametrization of density functional dispersion correction (DFT-D) for the 94 elements H-Pu," J. Chem. Phys. 132, 154104 (2010).

${ }^{26}$ S. Grimme, S. Ehrlich, and L. Goerigk, "Effect of the damping function in dispersion corrected density functional theory," J. Comput Chem. 32, 1456-1465 (2011).

${ }^{27}$ The basis sets for $\mathrm{H}, \mathrm{N}$, and $\mathrm{O}$ were not modified in the present work to keep results as much as possible consistent with original composite methods.

${ }^{28}$ R. Dovesi, A. Erba, R. Orlando, C. M. Zicovich-Wilson, B. Civalleri, L. Maschio, M. Rérat, S. Casassa, J. Baima, S. Salustro et al., "Quantum-mechanical condensed matter simulations with CRYSTAL," Wiley Interdiscip. Rev.: Comput. Mol. Sci. 8, e1360 (2018).

${ }^{29}$ C. Pisani, R. Dovesi, and C. Roetti, Hartree-Fock Ab Initio Treatment of Crystalline Systems, Lecture Notes in Chemistry Vol. 48 (Springer Verlag, Heidelberg, 1988).

${ }^{30}$ R. Orlando, M. De La Pierre, C. M. Zicovich-Wilson, A. Erba, and R. Dovesi, "On the full exploitation of symmetry in periodic (as well as molecular) self-consistent-field $a b$ initio calculations," J. Chem. Phys. 141, 104108 (2014).

${ }^{31}$ A. Erba, J. Baima, I. Bush, R. Orlando, and R. Dovesi, "Large-scale condensed matter DFT simulations: Performance and capabilities of the CRYSTAL code," J. Chem. Theory Comput. 13, 5019-5027 (2017).
${ }^{32}$ J. Rezác, K. E Riley, and P. Hobza, "S66: A well-balanced database of benchmark interaction energies relevant to biomolecular structures," J. Chem. Theory Comput. 7, 2427-2438 (2011).

${ }^{33}$ A. Otero-De-La-Roza and E. R. Johnson, "A benchmark for non-covalent interactions in solids," J. Chem. Phys. 137, 054103 (2012).

${ }^{34} \mathrm{~A}$. M. Reilly and A. Tkatchenko, "Seamless and accurate modeling of organic molecular materials," J. Phys. Chem. Lett. 4, 1028-1033 (2013).

${ }^{35}$ P. Hao, Y. Fang, J. Sun, G. I. Csonka, P. H. T. Philipsen, and J. P. Perdew, "Lattice constants from semilocal density functionals with zero-point phonon correction," Phys. Rev. B 85, 014111 (2012).

${ }^{36}$ M. F. Peintinger, D. V. Oliveira, and T. Bredow, "Consistent Gaussian basis sets of triple-zeta valence with polarization quality for solid-state calculations," J. Comput. Chem. 34, 451-459 (2013).

${ }^{37}$ Reference data adopted in the POB dataset were not back-corrected to estimate the athermal limit. Therefore, it should be expected that the computed equilibrium unit cell volumes are slightly underestimated with respect to experimental data, which is indeed the case for HFsol-3c, PBEsol-3c, and HSEsol-3c.

${ }^{38} \mathrm{~L}$. Spanu, S. Sorella, and G. Galli, "Nature and strength of interlayer binding in graphite,” Phys. Rev. Lett. 103, 196401 (2009).

${ }^{39}$ Y. Baskin and L. Meyer, "Lattice constants of graphite at low temperatures," Phys. Rev. 100(2), 544 (1955).

${ }^{40}$ G. Sansone, A. J. Karttunen, D. Usvyat, M. Schütz, J. G. Brandenburg, and L. Maschio, "On the exfoliation and anisotropic thermal expansion of black phosphorus," Chem. Commun. 54, 9793-9796 (2018).

${ }^{41}$ G. Sansone, L. Maschio, D. Usvyat, M. Schütz, and A. Karttunen, "Toward an accurate estimate of the exfoliation energy of black phosphorus: A periodic quantum chemical approach, "J. Phys. Chem. Lett. 7, 131-136 (2015).

${ }^{42}$ G. A. Lager, J. D. Jorgensen, and F. J. Rotella, "Crystal structure and thermal expansion of $\alpha-\mathrm{SiO}_{2}$ at low temperatures,” J. Appl. Phys. 53, 6751-6756 (1982).

${ }^{43}$ R. Tarumi, K. Nakamura, H. Ogi, and M. Hirao, "Complete set of elastic and piezoelectric coefficients of $\alpha$-quartz at low temperatures," J. Appl. Phys. 102, 113508 (2007).

${ }^{44}$ L. Levien, C. T Prewitt, and D. J. Weidner, "Structure and elastic properties of quartz at pressure," Am. Miner. 65, 920-930 (1980).

${ }^{45}$ C. M. Zicovich-Wilson, F. Pascale, C. Roetti, V. R. Saunders, R. Orlando, and R. Dovesi, "Calculation of the vibration frequencies of $\alpha$-quartz: The effect of Hamiltonian and basis set," J. Chem. Phys. 25, 1873-1881 (2004).

${ }^{46}$ E. Caldeweyher, C. Bannwarth, and S. Grimme, "Extension of the D3 dispersion coefficient model," J. Chem. Phys. 147, 034112 (2017).

${ }^{47}$ E. Caldeweyher, S. Ehlert, A. Hansen, H. Neugebauer, S. Spicher, C. Bannwarth, and S. Grimme, "A generally applicable atomic-charge dependent London dispersion correction," J. Chem. Phys. 150(15), 154122 (2019).

${ }^{48} \mathrm{~B}$. M. Axilrod and E. Teller, "Interaction of the van der Waals type between three atoms," J. Chem. Phys. 11, 299-300 (1943).

${ }^{49}$ Y. Muto, "Force between nonpolar molecules," J. Phys. Math. Soc. Jpn. 17, 629631 (1943). 